\title{
The Esophageal Anastomosis: How Improving Blood Supply Affects Leak Rate
}

\author{
Kevin M. Reavis
}

Received: 28 October 2008 / Accepted: 15 April 2009 / Published online: 5 May 2009

(C) The Author(s) 2009. This article is published with open access at Springerlink.com

Keywords Ischemic conditioning · Supercharge ·

Esophageal leak · Anastomosis · VEGF - Delay phenomenon

\section{Introduction}

Esophagogastric resection and anastomosis have long served as a technically demanding task for gastrointestinal and thoracic surgeons. The location of the structures involved and the morbidity incurred from ischemia of the completed conduit have put into question the validity of the operation. Several techniques including preoperative embolization of specified vessels, vascular anastomoses at the time of esophagogastric anastomosis, and staged vascular ligation followed by delayed esophagogastric resection and anastomosis have been employed over the last 50 years to reduce leak rates from $25 \%$ to less than $6 \%$ in some series. Angiogenic gene therapy is now being investigated as a modality to further reduce morbidity of the anastomosis. The next advancement in esophagogastric resection and anastomosis will likely utilize a multidisciplinary approach with endoscopic delivery of conditioning agents, as well as

This paper was originally presented as part of an SSAT/SAGES Joint Symposium entitled, "The Gastrointestinal Anastomosis: Evidence vs. Tradition; The Esophageal Anastomosis", at the SSAT 49th Annual Meeting, May 2008, in San Diego, California. The other article presented in this symposium was Raymond D, Traditional Methods to Prevent Leak.

K. M. Reavis $(\bowtie)$

Division of Gastrointestinal Surgery, Department of Surgery,

University of California, Irvine Medical Center,

333 City Boulevard West, Suite 850,

Orange, CA 92868, USA

e-mail: kreavis@uci.edu surgical preparation of the conduit in order to reduce morbidity and optimize patient outcomes.

\section{Discussion}

From the inception of surgical approaches to diseases of the human foregut, the resection and anastomosis of the esophagus has provided surgeons with a formidable challenge. Traversing the thoracic outlet into the posterior mediastinum, the esophagus courses between vital and fragile structures making the surgical approach to it (along with resection and replacement) a technically demanding and inherently risky endeavor.

From the 1670s through the initial portion of the twentieth century, surgeons sought to resect the esophagus and replace it either using non-autologous components such as eel skin or rubber hoses, or avoid the hazards of the posterior mediastinum for reconstruction via substernal or extracorporeal approaches. By the second half of the twentieth century, the stomach and colon became the standard conduits for use as the neoesophagus, most often placed in anatomic apposition to the transected end of the esophagus. The configuration of these reconstructions, which are predisposed to inadequate blood supply to the furthest portion of the conduits and the resultant ischemia, has played a role in the sequelae of esophagogastric and esophagocolonic anastomotic leaks in $3-25 \%$ of cases.

To combat ischemia of the neoesophagus, several approaches have been investigated with the common goals of improved blood flow to the at risk anastomosis and reduced rates of ischemia related anastomotic leaks. In the late 1990s, Akiyama et al. performed preoperative angiographic embolization of the left gastric, distal right gastric, 
and splenic arteries in a study group of patients, followed a few days later by esophagogastrectomy with gastric tubularization and esophagogastrostomy. The same operation was performed in a single stage in a control group without preoperative embolization. The reduction in blood flow to the furthest end of the gastric conduit was 33\% from baseline in the embolization group and 67\% from baseline in the control group. Anastomotic leak rate was slightly higher in the control group. The majority of embolized patients suffered either epigastric pain and/or nausea following embolization, however splenic infarction was a rare event. ${ }^{1}$

A different approach to reducing the effects of ischemia to the end of the gastric conduit was also being investigated via vascular anastomoses and "supercharging" of the neoesophagus. Although descriptions of mesenteric vascular augmentation commenced from the 1940s, quantitative investigation of this principle was performed by Nagawa et al. in 1997 when nine patients underwent intraoperative anastomoses between the left gastroepiploic and transverse cervical arteries resulting in no anastomotic leaks, as compared to the 10-25\% leak rate previously experienced by the group using unaugmented techniques. ${ }^{2}$ Murikami et al. expanded this experience to a group of 15 patients who underwent vascular anastomoses involving the short gastric vessels and transverse cervical vessels. A $36 \%$ increase in blood flow was noted at the tip of the gastric conduit following venous anastomosis, due to decreased venous congestion and a $108 \%$ increase in blood flow was noted following venous and arterial anastomoses. No leaks were noted in the study patients, while $23 \%$ of the 26 control patients suffered anastomotic leaks. ${ }^{3}$ The vascular anastomoses resulted in both decreased venous congestion and augmented arterial inflow at the level of the at risk anastomosis during the critical period of initial healing, and hence reduced the incidence of anastomotic leaks. This improved outcome however required multiple surgical teams with experience in microvascular anastomoses as well as esophageal surgery and increased operative time and logistic (microsurgical) arrangements. A simple approach which could be applied via a minimally invasive approach would streamline this process.

In 2004, Reavis et al. revisited the concept of preoperative ischemic conditioning in an opossum model. Fourteen animals underwent ligation of the left, distal right, and short gastric vascular pedicles 4 weeks prior to definitive esophagogastrectomy and esophagogastrostomy formation instituting the Delay phenomenon of conduit conditioning. Blood flow to the gastric fundus initially dropped by $73 \%$ following ligation; however, at the time of definitive resection blood flow to the newly formed gastric tube was three times higher in the Delay animals when compared to 15 control animals which underwent definitive resection without prior Delay. There were no leaks in the Delay group and two leaks leading to death in the control group. Upon histological evaluation, the anastomotic tissue showed significantly more atrophy of the muscularis mucosa in the control group than the Delay group and the Delay animals showed greater numbers of capillaries throughout the conditioned tissue when compared to the baseline control animals. ${ }^{4}$ Clinical use of Delay in humans was initiated by Nguyen et al. who staged nine individuals with laparoscopic ligation of the left gastric vascular pedicle along with a staging laparoscopy and placement of jejunal feeding catheter, followed by definitive esophagogastrectomy and esophagogastrostomy several days later. The additional procedure took $45 \mathrm{~min}$ and none of the patients leaked. ${ }^{5,6}$ Holscher et al. expanded this concept to 83 patients who underwent ligation of the left gastric vascular pedicle and gastric conduit formation followed by definitive resection and anastomosis 4 to 5 days later. Six percent of patients had minor leaks which were treated with stenting and there were no deaths at 3-months follow-up. ${ }^{7}$ The advantages of this concept include the simplicity of application via a minimally invasive approach, the opportunity to recover from two smaller operations rather than one single larger operation, and the reduction of clinical severity of anastomotic leaks when they do occur. Improving on this requires a single stage application with the most minimal invasive approach possible and very specifically targeted tissues. This has been studied using plasmid delivery of vascular endothelial growth factor (VEGF) by Enesvedt et al. Five study opossums received VEGF 165 to the gastric fundus involved in the most at risk portion of the neoesophagus immediately following esophagogastrectomy and esophagogastrostomy. There were no leaks in the study group and one leak in the control group of six animals. Increased bursting strength, neovascularization, and VEGF mRNA were detected in study animal tissue compared to the control group. ${ }^{8}$ This technology has the potential to be deployed laparoscopically, endoscopically, or even orally via cell receptor directed therapy and is applicable not only to esophageal anastomoses but to any hollow viscus anastomosis. At the present time this is probably best reserved for situations involving non-malignant disease states in order to avoid potentially augmenting blood flow to microscopic residual malignant disease following resection.

\section{Conclusions}

Overall, the use of various modalities to increase blood flow to the esophageal anastomosis following resection and reconstruction results in decreased leak rates from initially as high as $25 \%$ to now less than $6 \%$. In order to improve on the previous advances, we will likely employ a multidisci- 
plinary approach incorporating molecular and genetic engineers to develop targeted delivery systems and endoscopic as well as surgical delivery of those systems to the at risk tissue, in order to condition the new conduit and strive for optimal surgical outcomes.

Open Access This article is distributed under the terms of the Creative Commons Attribution Noncommercial License which permits any noncommercial use, distribution, and reproduction in any medium, provided the original author(s) and source are credited.

\section{References}

1. Akiyama S, Kodera Y, Sekiguchi H, et al. Preoperative embolization therapy for esophageal operation. J Surg Oncol. 1998;69 (4):219-223. doi:10.1002/(SICI)1096-9098(199812)69:4<219:: AID-JSO5>3.0.CO;2-7.

2. Nagawa H, Seto Y, Nakatsuka T, Kaizaki S, Muto T. Microvascular anastomosis for additional blood flow in reconstruction after intrathoracic esophageal carcinoma surgery. Am J Surg. 1997;173 (2):131-133. doi:10.1016/S0002-9610(96)00410-2.

3. Murakami M, Sugiyama A, Ikegami T, et al. Revascularization using the short gastric vessels of the gastric tube after subtotal esophagectomy for intrathoracic esophageal carcinoma. J Am Coll Surg. 2000;190(1):71-77. doi:10.1016/S1072-7515(99)00234-3.

4. Reavis KM, Chang EY, Hunter JG, Jobe BA. Utilization of the delay phenomenon improves blood flow and reduces collagen deposition in esophagogastric anastomoses. Ann Surg. 2005;241 (5):736-745. doi:10.1097/01.sla.0000160704.50657.32.

5. Nguyen NT, Longoria M, Sabio A, et al. Preoperative laparoscopic ligation of the left gastric vessels in preparation for esophagectomy. Ann Thorac Surg. 2006;81(6):2318-2320. doi:10.1016/j.athoracsur. 2005.05.033.

6. Varela JE, Reavis KM, Hinojosa MW, Nguyen NT. Laparoscopic gastric ischemic conditioning prior to esophagogastrectomy: technique and review. Surg Innov. 2008;15(2):132-135.

7. Enestvedt K, Winn SR, Diggs BS, et al. VEGF Gene Therapy Improves Anastomotic Healing in the Gastrointestinal Tract: Applications in Esophageal Surgery. Presented at Society for Surgery of the Alimentary Tract, May 21, 2008, San Diego.

8. Hölscher AH, Schneider PM, Gutschow C, Schröder W. Laparoscopic ischemic conditioning of the stomach for esophageal replacement. Ann Surg. 2007;245(2):241-246. doi:10.1097/01. sla.0000245847.40779.10. 